\title{
Degloving Injury of the Lower Extremity: Report of Two Cases
}

\author{
Alexandros Kyriakidis ${ }^{1}$, Ioannis Katsaros ${ }^{1}$, Evangelos Vafias ${ }^{1}$, Loukas Agorgianitis ${ }^{1}$, \\ Vladimiros Kyriakidis ${ }^{1}$, Athanasios Zacharopoulos ${ }^{2}$
}

${ }^{1}$ Department of General Surgery, General Hospital of Amfissa, Amfissa, Greece, ${ }^{2}$ Department of Orthopedic Surgery, General Hospital of Amfissa, Amfissa, Greece

Correspondence: gikats13@gmail.com

Tel.: + 306970801235

Fax.: + 302265022086

Received: 18 February 2019; Accepted: 18 September 2019

\begin{abstract}
Objective. The aim of our article is to highlight the importance of the immediate treatment of lower extremity degloving injuries, in order to prevent complications. Cases Presentation. Here we present two cases of degloving injury of the lower extremity, both resulting from motorway accidents. The first one concerned a 65-year-old man suffering from multiple limb fractures and a degloving injury of the right thigh, which was immediately treated with extensive debridement and primary full-thickness skin graft re-approximation. The second case involved a 63-year-old woman who presented with cervical vertebrae fractures and a degloving injury of the left posterior leg, which, due to the severity of her condition, was treated with a delayed approach resulting in skin necrosis, which required surgical debridement, alginate dressing and foam cover. Conclusions. The optimal approach to treatment of degloving injuries is challenging and they warrant immediate surgical attention. An early diagnosis and the evaluation of tissue viability are important in order to prevent limb-threatening situations.
\end{abstract}

Key Words: Degloving Trauma - Tissue Injury • Skin - Reconstructive Surgery.

\section{Introduction}

Degloving injuries are when skin or underlying tissues are stripped from the respective bone structures. They are usually a result of traumatic road accidents, wringer or industrial roller injuries. The lower and upper extremities are the most common sites $(1,2)$.

The skin often remains intact and the limb assimilates a "fluid-containing bag", due to the presence of an extensive hematoma between the skin and the underling fascia (compartment syndrome) (3). In the case of skin damage, a full-thickness skin graft is observed (4). In both cases the large regional vessels may be injured, resulting in capillary bed impairment (5). Edema formation also plays a very important role, as it increases the tissues' oxygen demands in order to diffuse the functioning capillaries $(6,7)$. These incidents warrant proper surgical management in order to prevent massive sloughing.

The objective of our article is to point out the necessity of a prompt approach to these injuries in order to secure the viability of the lower limb and prevent complications.

\section{Cases Presentation}

Presentation of both cases was conducted according to the CARE Guidelines (Consensus-based Clinical Case Reporting Guideline Development) and the patients' informed consent was obtained (8). 


\section{Case 1}

A 65 year-old male patient was admitted to our hospital following a high-speed road accident on his motorbike. He was suffering from an open (grade I) fracture of his right distal radius and ulna, a right femoral diaphyseal fracture, as well as a large degloving injury of his right thigh (Figure 1). The injured lower limb presented in external rotation and was shorter than the other one. The degloving injury covered a triangular region extending from the perineum (medially) to the greater trochanter (laterally) and the middle of the anterior surface of the femur (inferiorly). It was accompanied by edema and erythema, but there was no active bleeding.

The patient was immediately transferred to the operating theater. Under general anesthesia extensive debridement of the injured lower limb took place, by removing the subcutaneous fat from the damaged skin and applying it as a free graft. The femoral fracture was stabilized using external fixation. Furthermore, the right forearm fracture was also stabilized utilizing an external fixation. During a 2-month follow-up period, the pa-

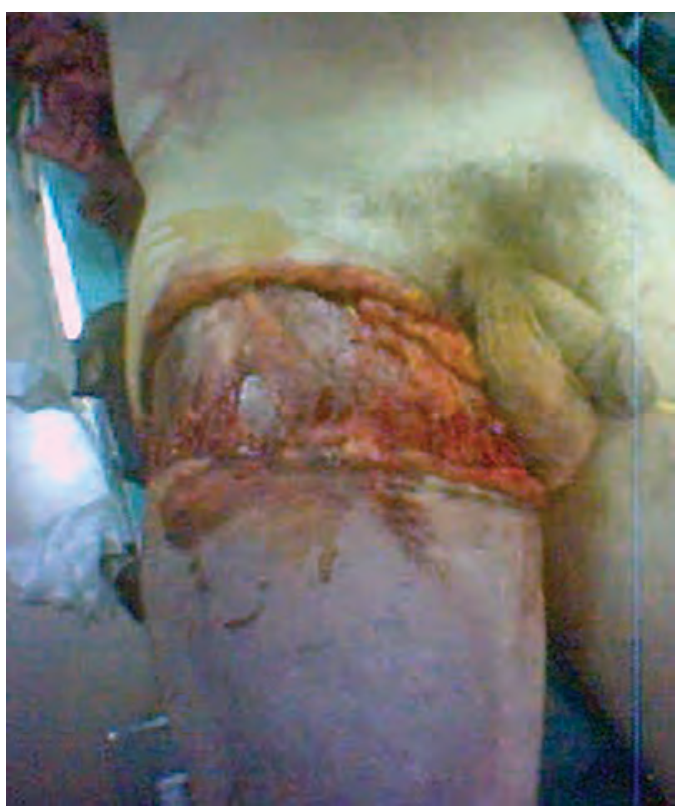

Figure 1. Degloving injury of the right thigh. tient showed no signs of infection, no skin necrosis at the degloving injury site, and the patient was able to move using a cane.

\section{Case 2}

A 63-year-old female patient was transferred to our emergency department after a car accident. She suffered from multiple fractures of the C6 and C7 vertebral bodies and spinous processes, and an extensive degloving injury of the left posterior leg extending from the popliteal fossa to the middle of the gastrocnemius muscles (Figure 2a). Additionally, hematomas of the left scapular region and the left temporal region were recorded.

The patient was initially evaluated and treated for her cervical and cranial injuries. Following that (after five hours), the lower limb wound was approximated using simple sutures. After three days, full-depth dermal necrosis at the site of the degloving injury was noted and thorough surgical debridement followed. The patient was treated after-

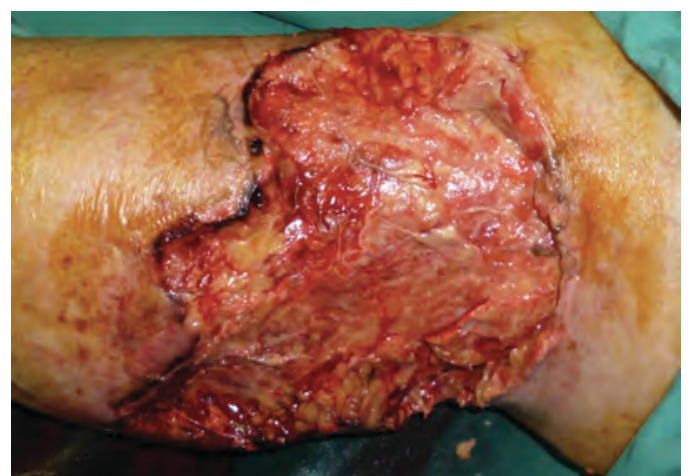

Figure 2a. Degloving Injury of the Posterior Left Leg.

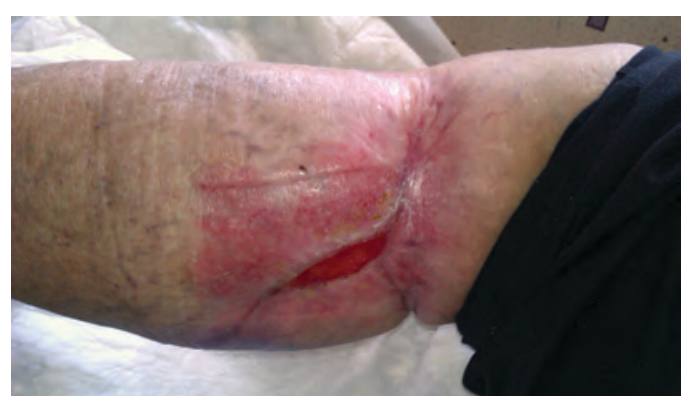

Figure 2b. Degloving Injury of the Posterior Left Leg -3 months following treatment. 
wards using an alginate dressing and foam cover. After a three-month follow-up period the degloving injury was almost completely healed (Figure $2 \mathrm{~b}$ ).

\section{Discussion}

Skin is the most extensive organ of the human organism and plays a multifunctional role (excretory, protective, sensory and temperature regulation). It can be damaged in multiple ways, including direct trauma, stretching, degloving and undermining during an operation (9). In the case of a firm and violent pull, skin may be peeled back over the underlying tissues, resulting in degloving injuries (9).

Depending on the extent of the damage, a wide variety of reconstructive surgical techniques are available for tissue repair. Yan et al. propose three patterns of degloving injury (a. purely degloving injury, b. degloving injury with involvement of deep soft tissues, c. degloving injury with deep bone fractures) and adjust their treatment approach accordingly (10). An immediate full-thickness skin graft after an extensive debridement is considered a feasible treatment approach even in elderly patients, in the case of non-extensive injuries $(10,11)$. If the skin is intact, it can be de-fatted and reapplied immediately as a full thickness graft (12). In the case of skin damage, split skin grafts can be retrieved and used immediately if the wound site is suitable. These grafts can also be stored for a secondary procedure 1 to 2 weeks following the injury. Securing or even restoring the extremity's sensibility is a challenge for the operating team. Suturing injured nerves can be tricky, and even when careful primary nerve anastomosis is achieved, the results are often unsatisfactory, probably as a result of the mechanism of the nerve injury (13).
As far as soft tissue elements are concerned, an initial evaluation of their viability is crucial. This will guide the debridement and determine whether primary closure or immediate reconstruction are feasible. Direct observation is always a useful tool in this direction. Skin with rapid capillary refill, bleeding edges and good colour is most likely to be viable. It is therefore recommended that thorough debridement of all non-perfused, non-viable tissues be performed immediately, in order to avoid the development of sepsis. Any damaged muscles should be resected to prevent the sequelae of severe myonecrosis or acute tubular necrosis. Denuded areas should be temporarily covered with either moistened dressings, xenografts or both. Reconstructive procedures, if needed, should be guided by the condition of local tissues. The treatment goal should be wound coverage with a pliable, sensitive, and cosmetically similar tissue that will allow early mobilization. In the case of an open injury, all the appropriate protective measures must be taken in order to prevent serious life-threatening infections, such as tetanus and gas gangrene (4).

\section{Conclusions}

Degloving injuries are very serious and potentially limb-threatening soft tissue lesions. They are usually the result of high-energy accidents. They may impair the skin and underlying soft tissues, rendering the human organism defenseless and vulnerable to soft-tissue necrosis. The treatment approach depends on the time of diagnosis and the extent of tissue injury. In the early stages and with good soft-tissue condition, immediate re-application of a full thickness graft is the treatment of choice. In later stages, a more reconstructive approach should be followed in order to counter contamination and inflammation of the surrounding tissue. 


\section{What Is Already Known on this Topic:}

Degloving injuries involve skin and underlying tissues being stripped from their respective bone structures. Various techniques have been described for their treatment extending from initial debridement and full thickness graft re-appliance to extensive reconstructive operations.

\section{What this Case Adds:}

An immediate surgical approach is vital in order to secure the optimal functional and aesthetic result to the affected area. A delayed approach can lead to limb-threatening complications.

Authors' Contributions: Conception and design: $\mathrm{AK}$ and $\mathrm{AZ}$; Acquisition, analysis and interpretation of data: IK, EV and LA; Drafting the article: IK, EV and LA; Revising it critically for important intellectual content: AK, IK and AZ; Approved final version of the manuscript: AK, IK, EV, LA, VK and AZ.

Conflict of Interest: The authors declare that they have no conflict of interest.

\section{References}

1. Khan AT, Tahmeedullah, Obaidullah. Degloving injuries of the lower limb. J Coll Physicians Surg Pak. 2004;14(7):416-8.

2. Lim SM, Lee ST. The pattern and management of traumatic soft tissue injuries of the lower limb. Ann Acad Med Singapore. 1982;11(2):145-53.

3. Waikakul S. Revascularization of degloving injuries of the limbs. Injury. 1997;28(4):271-4.

4. Yuan K, Zhao B, Cooper T, Jin Z, Zhou X, Chen X, et al. The management of degloving injuries of the limb with full thickness skin grafting using vacuum sealing drainage or traditional compression dressing: A comparative cohort study. J Orthop Sci. 2019.
5. Graf P, Biemer E. Degloving injuries of the soft tissues of the heel. An indication for microvascular revascularization! [in German]. Chirurg. 1994;65(7):642-5.

6. Huemer GM, Schoeller T, Dunst KM, Rainer C. Management of a traumatically avulsed skin-flap on the dorsum of the foot. Arch Orthop Trauma Surg. 2004;124(8):559-62.

7. Yang SC, Su JY, Yu SW, Tu YK. Retrograde tibial nail for femoral shaft fracture with severe degloving injury. Chang Gung Med J. 2004;27(6):454-8.

8. Gagnier JJ, Kienle G, Altman DG, Moher D, Sox $\mathrm{H}$, Riley D, et al. The CARE guidelines: consensusbased clinical case reporting guideline development. BMJ Case Rep. 2013;2013.

9. Weinand C, Prommersberger KJ, Hahn P, Giunta RE, Krimmer H. Strategy for defect coverage in extensive degloving and crush injuries of 4 fingers [in German]. Handchir Mikrochir Plast Chir 2000;32(6):424-9.

10. Yan H, Gao W, Li Z, Wang C, Liu S, Zhang F, et al. The management of degloving injury of lower extremities: technical refinement and classification. J Trauma Acute Care Surg. 2013;74(2):604-10.

11. Jeng SF, Hsieh CH, Kuo YR, Wei FC. Technical refinement in the management of circumferentially avulsed skin of the leg. Plast Reconstr Surg. 2004;114(5):1225-7.

12. Lin GT. Bone resorption of the proximal phalanx after tendon pulley reconstruction. J Hand Surg Am. 1999;24(6):1323-6

13. Muneuchi G, Suzuki S, Ito O, Saso Y. One-stage reconstruction of both the biceps brachii and triceps brachii tendons using a free anterolateral thigh flap with a fascial flap. J Reconstr Microsurg. 2004;20(2):139-42. 